\title{
SÍNTESIS Y CARACTERIZACIÓN MORFOLÓGICA DE NANOTUBOS DE ZNO SOBRE SUBSTRATOS DE VIDRIO Y DE $\mathrm{SNO}_{2}: \mathrm{F}$
}

\author{
SYNTHESIS AND MORPHOLOGICAL CHARACTERIZATION OF \\ ZNO NANOTUBES ON SUBSTRATES OF GLASS AND $\mathrm{SNO}_{2}: \mathrm{F}$
}

\author{
Julio Alarcón ${ }^{1}$, Javier Gago ${ }^{2}$, Juan Rodríguez ${ }^{3}$
}

\section{RESUMEN}

En el presente trabajo se reporta la sintesis y caracterización morfológica de nanotubos de $\mathrm{ZnO}$, fabricados a partir de semillas de óxido de zinc fabricadas por el método del rociado pirolítico. El crecimiento de los nanotubos de $\mathrm{ZnO}$ se llevó a cabo sumergiendo los sustratos con las semillas de $\mathrm{ZnO}$ en una solución acuosa de nitrato de zinc y metenamina. Las muestras fabricadas bajo diferentes condiciones se analizaron en su morfología por Microscopia Electrónica de Barrido.

Palabras clave.- Fotocatálisis, ZnO, Nanotubos.

\begin{abstract}
In this paper the synthesis and morphologic characterization of $\mathrm{ZnO}$ nanotubes, made from zinc oxide seed produced by the spray pyrolysis method is reported. The growth of nanotubes of $\mathrm{ZnO}$ was conducted by dipping the substrate with a $\mathrm{ZnO}$ seed aqueous solution of zinc nitrate and methenamine. The samples manufactured under different conditions were analyzed in morphology by Scanning Electron Microscopy.
\end{abstract}

Key words.- Photocatalysis, ZnO, Nanotubes.

\section{INTRODUCCION}

La necesidad de nuevos materiales que tengan forma de degradar de manera eficiente contaminantes en medio líquido son temas de profundo interés mundial [1]. Varios fotocatalizadores, especialmente los del tipo metal óxido como el óxido de titanio, óxido de estaño y el óxido de zinc, son materiales promisorios para la degradación de contaminantes orgánicos mediante la utilización de luz UV o solar [2-9]. El óxido de zinc $(\mathrm{ZnO})$, un semiconductor tipo-n de ancha banda prohibida, juega un importante papel en muchos campos de aplicación desde la optoelectrónica hasta la conversión de energía, fotocatálisis y sensores de gas [10-14]. En cuanto a la fotocatálisis, el $\mathrm{ZnO}$ es más eficiente que el $\mathrm{TiO}_{2}$, el fotocatalizador más intensamente estudiado, en la fotodegradación de algunos compuestos orgánicos [15-19].

Sin embargo, la aplicación del $\mathrm{ZnO}$ como fotocatalizador fue muy limitada debido a su fotoinestabilidad en solución acuosa. Por lo tanto, mejorar la estabilidad del $\mathrm{ZnO}$ sin sacrificar su eficiencia representa problemas científicos y tecnológicos muy atractivos por resolver.

La Revista Científica TECNIA protege los derechos de autor bajo la Licencia 4.0 de Creative Commons: Attribution 4.0 International (CC BY 4.0)

${ }^{1}$ Consultor en Euroequip, ${ }^{2}$ Maestro de la Dirección de Investigación y Desarrollo del Instituto Peruano de Energía Nuclear - IPEN, ${ }^{3}$ Dr. de la Facultad de Ciencias de la Universidad Nacional de Ingeniería, Director del Instituto General de Investigación de la Universidad Nacional de Ingeniería. 
Kislov et al., investigaron la fotoestabilidad y la actividad fotocatalítica de diferentes superficies cristalinas de $\mathrm{ZnO}$ [20].

Ellos demostraron que la fotólisis del $\mathrm{ZnO}$ dependía fuertemente de la orientación, y que la superficie (000-1)-O presentaba la más baja estabilidad en la fotólisis. Por otra parte, la reacción fotocatalítica de la superficie (10-10) presenta la más alta actividad seguida de la superficie, (0001)-Zn, mientras que la superficie (000-1)-O muestra la más baja actividad. Basados en esta investigación, el reto es diseñar micro/nano estructuras de $\mathrm{ZnO}$ que presenten superficies fotoestables y encontrar métodos para bloquear la superficie polar con terminando $-\mathrm{O}$, lo que haría al $\mathrm{ZnO}$ un material más resistente a la fotocatálisis.

De acuerdo a este análisis, las estructuras tubulares de $\mathrm{ZnO}$ orientadas en el eje c, podrían ser los candidatos idóneos para aplicaciones fotocatalíticas porque tales estructuras tubulares están compuestos principalmente de superficies estables (10-10) en vez de superficies (000-1)-O y (0001)-Zn y poseen una gran área superficial. Las principales técnicas usadas para el crecimiento de nanotubos de $\mathrm{ZnO}$ incluyen el crecimiento asistido en plantillas y electrodeposición [21-25]. Aunque estos métodos pueden producir nanotubos de $\mathrm{ZnO}$ de alta calidad, generalmente enfrentan los problemas de remover las plantillas, tediosos procedimientos de operación, o requerimiento de sustratos especiales. Recientemente, se ha mostrado interés por el proceso de deposición por baño químico (CBD) para el crecimiento de varias estructuras tubulares de $\mathrm{ZnO}$ debido a que se requiere temperaturas relativamente bajas y es sencillo de manipular en diferentes sustratos [26].

\section{EXPERIMENTAL}

En este trabajo se utilizaron químicos (Merck) P.A. Se usaron vidrio simple y vidrio FTO $\left(\mathrm{SnO}_{2}: \mathrm{F}\right)$ como sustratos. Las semillas de $\mathrm{ZnO}$ se prepararon por el método del rociado pirolítico, en el cual, básicamente, se prepara una solución de acetato de zinc $0.1 \mathrm{M}$ diluido en una relación de etanol-agua de $3: 1$, respectivamente, que actuarán como solventes. Esta relación etanol-agua se utilizó porque en un estudio previo se determinó que películas fabricadas bajo esta relación en volumen presentan una morfología más uniforme y tamaño de particula menor. Luego se agrega ácido acético para variar el $\mathrm{pH}$ a 4.0. La temperatura de deposición en el rociado pirolítico fue de $350^{\circ} \mathrm{C}$.

El crecimiento de los nanotubos de $\mathrm{ZnO}$ se llevó a cabo sumergiendo los sustratos con las semillas de $\mathrm{ZnO}$ en una solución acuosa equimolar de $0.1 \mathrm{M}$ de nitrato de zinc $\mathrm{Zn}(\mathrm{NO} 3) 2$ y $0.1 \mathrm{M}$ de metenamina (C6H12N4 HMT) a $75^{\circ} \mathrm{C}$ por 3 horas. Se varió el pH de esta solución agregando ácido acético.

Posteriormente, el sustrato fue removido de la solución, enjuagado con agua desionizada y sumergido nuevamente en una solución acuosa 0.3 $\mathrm{M}$ de $\mathrm{NaOH}$ a $80^{\circ} \mathrm{C}$ por 1 hora. Finalmente, se lavó el sustrato con agua desionizada y se secó a $100^{\circ} \mathrm{C}$ por una hora, ver Fig. 1. 


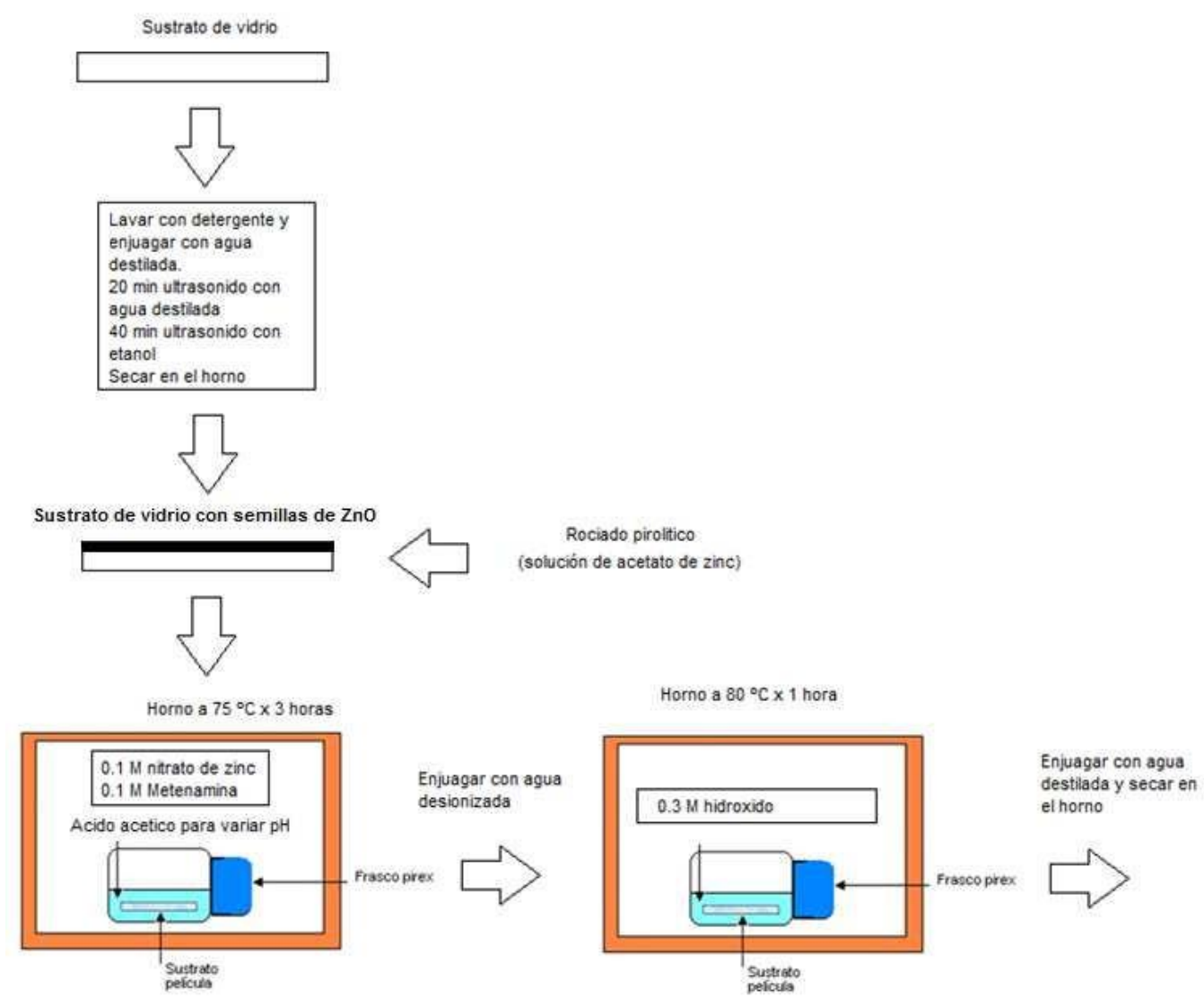

Fig. 1 Esquema de preparación de los nanotubos de ZnO.

\section{MORFOLOGÍA DE LAS SEMILLAS DE ZnO}

Se hicieron crecer los nanotubos sobre sustratos de vidrio simple y FTO, según Figuras 2 y 3 respectivamente.

Las condiciones en ambos casos fueron las mismas cambiando solo el tipo de sustrato.

Solución precursora de acetato de zinc $0.1 \mathrm{M}$ en una relación 3/1 etanol/agua, $\mathrm{pH} 4$ y la temperatura de depósito fue $350^{\circ} \mathrm{C}$. Se observa una distribución de semillas más homogénea en el sustrato FTO.

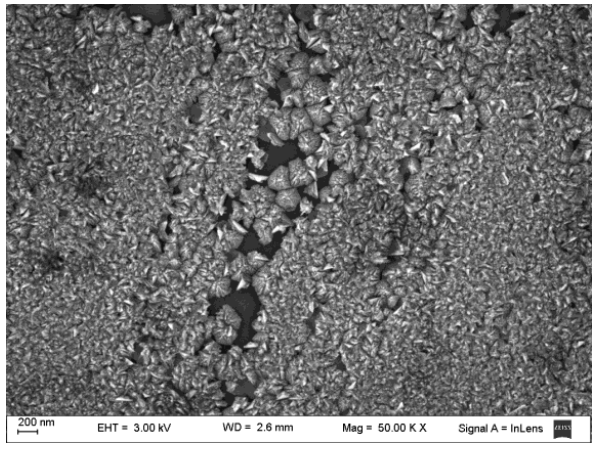

Fig. 2 Semillas de $\mathrm{ZnO}$ depositadas sobre un sustrato de vidrio simple. 


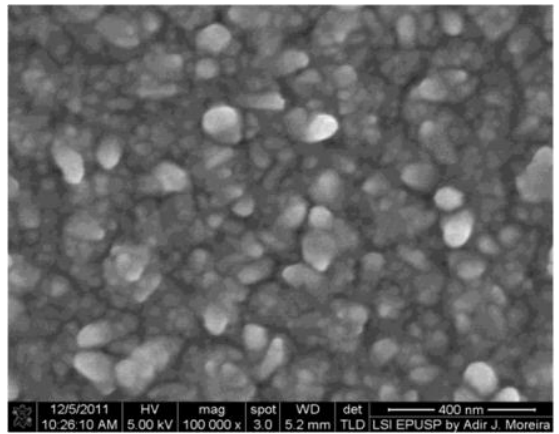

Fig. 3 Semillas de $\mathrm{ZnO}$ crecidas sobre un sustrato conductor transparente FTO $\left(\mathrm{SnO}_{2}: \mathrm{F}\right)$.

\section{MORFOLOGÍA DE LOS NANOTUBOS DE ZnO CRECIDOS}

La Figura 4 muestra los nanotubos de $\mathrm{ZnO}$ a partir de semillas de $\mathrm{ZnO}$ crecidas en la solución equimolar $0.1 \mathrm{M}$ de metenamina y nitrato de zinc como función del $\mathrm{pH}$ de esta solución equimolar. Este crecimiento se llevó a cabo en un horno a $75^{\circ} \mathrm{C}$ durante tres horas a $\mathrm{pH} 5.74$ y luego de un baño con $\mathrm{NaOH}, 0.3 \mathrm{M}$ por una hora a $80{ }^{\circ} \mathrm{C}$.

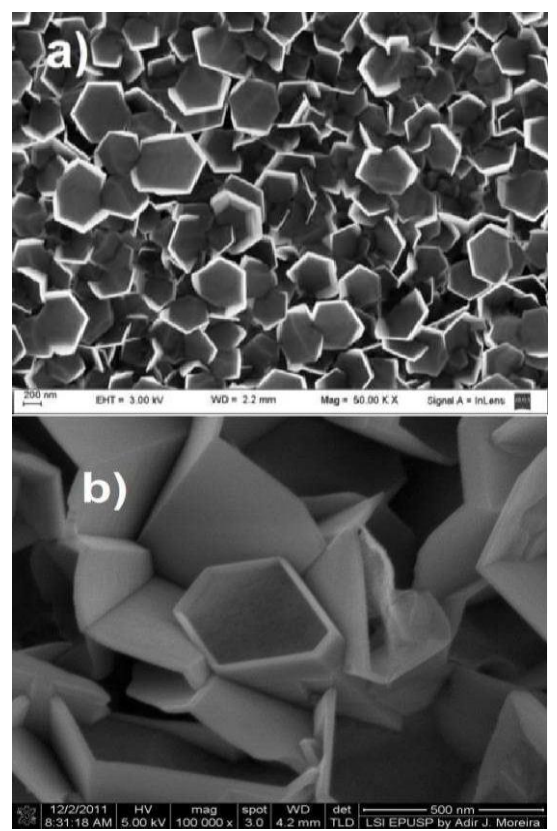

Fig. 4 Solución equimolar $0.1 \mathrm{~m}$ de metenamina y nitrato de zinc pH 5.74 a) Sustrato simple b) Sustrato FTO $\left(\mathrm{SnO}_{2}: \mathrm{F}\right)$.

\section{CONCLUSIONES}

Se han fabricado nanotubos de $\mathrm{ZnO}$ en baño químico de metenamina y nitrato de zinc a $\mathrm{pH} 5.74$, seguido por el baño químico en $\mathrm{NaOH}$. Los arreglos de nanotubos muestran una alta orientación perpendicular al sustrato, formándose nanotubos hexagonales con un espesor de pared de varias decenas de nanómetros y diámetros externos alrededor de $650 \mathrm{~nm}$. Debido a que los nanotubos crecieron a partir de las semillas de $\mathrm{ZnO}$, el tamaño de los nanotubos estará determinado por las semillas originales, las cuales pueden ser controladas variando las condiciones de su depósito en el rociado pirolítico, ese trabajo se encuentra en desarrollo.

\section{AGRADECIMIENTOS}

Este trabajo ha sido posible gracias a los proyectos FINCYT PIBAP 140-2013, proyecto PROCYT 2262008 -CONCYTEC-OAJ. Se agradece a la Dra. Claudia Marchi de la Universidad de Buenos Aires por la caracterización morfológica de las muestras.

\section{REFERENCIAS}

1. Fujishima, A., Honda, K., "Electrochemical Photolysis of Water at a Semiconductor Electrode". Nature 1972, 238, 37-38.

2. Wang, Z., Huang, B., Dai, Y., Qin, X., Zhang, X., Wang, P., Liu, H., Yu, J., "Highly Photocatalytic $\mathrm{ZnO} / \mathrm{In}_{2} \mathrm{O}_{3}$ Heteronanostructures Synthesized by a Coprecipitation Method". J. Phys. Chem. C 2009, 113, 4612-4617.

3. Yamashita, H., Honda, M., Harada, M., Ichihashi, Y., Anpo, M., Hirao, T., Itoh, N., Iwamoto, N., "Nanotubes include templateassisted growth and electro J. Phys". Chem. B 1998, 102, 10707-10711.

4. Quan, Quan, X., Yang, S., Ruan, X., Zhao, H., "Preparation of titnia nanotubes and theri enviromental applications as electrode, Environ". Sci. Technol . 2005, 39, 3770-3775.

5. Usseglio, S., Damin, A., Scarano, D.; Bordiga, S., Zecchina, A., Lamberti, C., "(I2)n encapsulation inside TiO2: a way to tune photoactivity in the visible region J. Am. Chem". Soc. 2007, 129, 2822-2828. 
6. Soli Casados, D., Vigueras-Santiago, E., Hernadez-Loez, S., Camacho-Loez, M. A., "Characterization and Photocatalytic Performance of Tin Oxide". Ind. Eng. Chem. Res. 2009, 48, 1249-1252.

7. Hou, L. R., Yuan C. Z, Peng Y., "Synthesis and photocatalytic property of $\mathrm{SnO}_{2} / \mathrm{TiO}_{2}$ nanotubes composites". Journal of Hazardous Materials. 2007; 139(2):310-315.

8. El-Maghraby, E. M., Nakamura, Y., Rengakuji, S., "Composite $\mathrm{TiO} 2-\mathrm{SnO} 2$ nanostructured films prepared by spin-coating with high photocatalytic performance". Catal. Commun. 2008, 9, 2357-2360.

9. Ohsaki, H., Kanai, N., Fukunaga, Y., Suzuki, M., Watanabe, T., Hashimoto, K., "Photocatalytic Properties of $\mathrm{SnO} 2 / \mathrm{TiO} 2$ Multilayers". Thin Solid Films 2006, 502, 138142.

10. Baxter, J. B., Aydil, E. S., "Dye-sensitized solar cells based on semiconductor morphologies with $\mathrm{ZnO}$ nanowires. Sol". Energy Mater Sol. Cells, 90 (2006) 607-622.

11. Yuas B. D., Yang P. D., "Nanowire-based alloxide solar cells, J. Am. Chem”. Soc. 2009, 131, 3756-3761.

12. Yamazaki, T., Wada, S., Noma, T., Suzuki, T., "Gas-Sensing Properties of Ultrathin Zinc Oxide Films". Sens. Actuators, B 1993, 13-14, 594-595.

13. Ye, C., Bando, Y., Shen, G., Golberg, D., "Thickness-Dependent Photocatalytic Performance of ZnO Nanoplatelets". J. Phys. Chem. B 110[31] (2006) 15146-15151.

14. Han, X.-G., He, H.-Z., Kuang, Q., Zhou, X., Zhang, X.-H., Xu, T., Xie, Z.-X., Zheng, L.S., "Controlling Morphologies and Tuning the Related Properties of Nano/Microstructured ZnO Crystallites". J. Phys. Chem. C 2009, 113, 584-589.

15. Sakthivel, S., Neppolian, B., Shankar, M. V., Arabindoo, B., Palanichamy, M., Murugesan, V. "Solar photocatalytic degradation of azo dye: comparison of photocatalytic efficiency of $\mathrm{ZnO}$ and TiO2". Sol. Energy Mater. Sol”. Cells 2003, 77, 65-82.

16. Yan, H., Hou, J., Fu, Z. \& Yang, B., Yang, P., Liu, K., Wen, M., Chen, Y., Fu, S., Li, F. "Growth and photocatalytic properties of one- dimensional $\mathrm{ZnO}$ nanostructures prepared by thermal evaporation. Mater". Res. Bull., vol. 44, 2009, p.1954-1958.

17. Pardeshi, S. K., Patil A. B., "Solar photocatalytic degradation of resorcinol a model endocrine disrupter in water using zinc oxide". Pardeshi, S. K.; Patil, A. B. J. Hazard. Mater. 2009, 163, 403-409.

18. Tian, J., Chen, L., Yin, Y., Wang, X., Dai, J., Zhu, Z., Liu, X., Wu, P., Surf., "Photocatalyst of $\mathrm{TiO}_{2} / \mathrm{ZnO}$ nano composite film: preparation, characterization, and photodegradation activity of methyl orange Coat". Technol. 2009, 204, 205-214.

19. Pardeshi, S. K., Patil, A. B., "Effect of morphology and crystallite size on solar photocatalytic activity of zinc oxide synthesized by solution free mechano-chemical method". J. Mol. Catal. A: Chem 2009, 308, 32-40.

20. Kislov, N., Lahiri, J., Verma, H., Goswami, D. Y., Stefanakos, E., Batzill, M., "Photocatalytic degradation of methyl orange over single crystalline $\mathrm{ZnO}$ : orientation dependence of photoactivity and photostability of ZnO”. Langmuir 2009, 25, 3310-3315.

21. Elias, J., Tena-Zaera, R., Wang, G.-Y., LevyClement, C., "Conversion of $\mathrm{ZnO}$ nanowires into nanotubes with tailored dimensions". Chem. Mater. 2008, 20, 6633-6637.

22. Guang-Wei, S., Xiao-Hong, Z.; Wen-Sheng, S.; Xia, F., Jack, C. C., Chun- Sing, L., ShuitTong, L., Chang-Hong, L., "Controlled synthesis of oriented single-crystal $\mathrm{ZnO}$ nanotube arrays on transparent conductive substrates". Appl. Phys. Lett. 2008, 92, 053111.

23. She, G. W., Zhang, X. H., Shi, W. S., Fan, X., Chang, J. C., Lee, C. S., Lee, S. T., Liu, C. H., "Controlled synthesis of oriented singlecrystal $\mathrm{ZnO}$ nanotube arrays on transparent conductive substrates". Appl. Phys. Lett. 2008, 92, 053111.

24. Yu, L. G., Zhang, G. M., Li, S. Q., Xi, Z. H., Guo, D. Z., "Fabrication of arrays of zinc oxide nanorods and nanotubes in aqueous solution under an external voltage". J. Cryst. Growth 2007, 299,184-188. 
25. Sun, Y., Fuge, G. M.; Fox, N. A., Riley, D. J., Ashfold, M. N. R., "Synthesis of aligned arrays of ultrathin $\mathrm{ZnO}$ nanotubes on a $\mathrm{Si}$ wafer coated with a thin ZnO film Adv". Mater. 2005, 17, 2477-2481.

26. Vayssieres, L., Keis, K., Hagfeldt, A., Lindquist, S. E., "Three-dimensional array of highly oriented crystalline $\mathrm{ZnO}$ microtubes Chem". Mater. 2001, 13, 4395-4398.

Correspondencia: jrodriguez@uni.edu.pe

Recepción de originales: abril 2015

Aceptación de originales: julio 2015 